\title{
FCSIT WhatsApp Chatbot
}

\section{TEO KUO HONG \& MOHAMAD JOHAN AHMAD KHIRI*}

\author{
Faculty of Computer Science and Information Technology, Universiti Malaysia Sarawak, 94300 Kota \\ Samarahan, Sarawak, Malaysia \\ *Corresponding authors: akmjohan@unimas.my
}

\begin{abstract}
WhatsApp is currently one of the most widely used communication mediums among students in tertiary education in the form of both personal messages and group messaging chats. Currently, the practice in most Malaysian Universities is that students need to consult the faculty staff or search through the faculty's website to obtain information related to their academic studies. However, the process of obtaining needed information can take some time ranging from minutes to hours and at times are not consistent as the queries are answered by different staff members. There are WhatsApp chat groups for students to interact with faculty staff, but the chat group can be overwhelmed by questions due to the huge number of group members hence causing important messages to be overlooked. In an attempt to overcome these challenges, we developed a FCSIT (Faculty of Computer Science and Information Technology) WhatsApp chatbot to solve these problems by offering a centralized platform of communication for both faculty staff and students to carry out information sharing through WhatsApp messages. Communication is in the form of personalized messages between the chatbot and the user thus solving the problem of overwhelming messages posed by group chats. Agile Kanban methodology was used to develop the system which promotes the use of a Kanban board to visualize the development stages. A questionnaire was distributed to lecturers, faculty staff and students to test the system and to gather opinions and suggestions from targeted users, which is the FCSIT community, in order to assess whether the system fulfils its objectives.
\end{abstract}

Keywords: Chatbot, Kanban Methodology, Natural Language Processing, WhatsApp

Copyright: This is an open access article distributed under the terms of the CC-BY-NC-SA (Creative Commons Attribution-NonCommercial-ShareAlike 4.0 International License) which permits unrestricted use, distribution, and reproduction in any medium, for non-commercial purposes, provided the original work of the author(s) is properly cited.

\section{INTRODUCTION}

WhatsApp is an instant messaging application which serves the purpose of replacing SMS (short message service) with a cross-platform mobile messenger application that works whenever devices are connected to the internet (Yeboah \& Ewur, 2014). In the university setting, WhatsApp is widely used as an informal communication between students for discussions about various projects or assignments, as well as information sharing among lecturers, faculty staff and students (Joicy \& Sornam, 2018). In order to further improve the efficiency of the current workflow being employed in the faculty, we leveraged the use of new technology such as the chatbot application. A chatbot is used to simulate human conversation in the form of text and sound to reply to a user query. Companies nowadays such as Spotify and Sephora utilize the chatbot as their virtual assistant to answer customers' queries and learn the behaviour of every customer based on conversations from time to time (Kim, 2018). The presence of a chatbot supports the automation of replies for similar queries, allowing human resources to focus on more important and qualitative tasks while improving the business with the least effort. Reply to queries can be received within seconds and is no longer restricted to office hours.

This project integrates both the use of WhatsApp which is a popular communication tool among staff members and student and a chatbot by creating a FCSIT (Faculty of Computer Science and Information Technology) WhatsApp chatbot application to support the teaching and learning ecosystem. The chatbot serves the purpose of providing a centralized communication platform to answer queries and delivering messages to users via the FCSIT WhatsApp chatbot. This project also includes the development of a website that provides a medium for the faculty administrative staff and lecturers to create the knowledge base for the chatbot, such as 